\title{
Research on Balance Measurement System Based on Embedded OMAP and Filtering Technology
}

\author{
Y. Jiao, G. F. Chen, Y. J. Wang \\ School of Electronic \& Information Engineering \\ Changchun University of Science and Technology \\ Changchun 130022, China
}

\begin{abstract}
At present, Micro controller and peripheral devices concatenated balance measurement system, which is in low integration level and large measurement error, and have a lot of obstacles to implement intelligent milling on the high speed occasions. Firstly, this paper discussed the way to implement intelligence milling balance measurement system based on embedded (Open Multimedia Application Platform, OMAP), OMAP dual-core architecture could reasonably plan the general task of the balance test system according to its advantages, and used ARM subsystem to complete control function of the balance mechanical's electrical system; DSP subsystem realized digital signal processing and calculation of unbalance. Secondly, in order to accurately extracted the characteristics of unbalanced signal from strong interference sources and effectively restrain signal interference, the software designed and completed tracking digital band pass filter based on harmonic wavelet package. Used the harmonic wavelet packet with the feature of infinite segmentation for the signal in the frequency domain to extract the characteristics of any period of weak signal. The simulation results showed that the digital band pass tracking filter based on harmonic wavelet packet can effectively restrain the nearly frequency signal interference, improve the signal-tonoise ratio, restore the initial unbalance signal characteristics. In high-speed situations (15000RPM above), the system can achieve high efficiency, high precision and intelligence targets.
\end{abstract}

Keywords-OMAP embedded system; harmonic wavelet packet; tracking filter; near-frequency interference

\section{INTRODUCTION}

The development of balancing machine depends on rotating machinery [1] and the improving balance theory. The core of rotating machinery is the rotating part (i.e. rotor), and the source of vibration and noise is related to the rotating part. Therefore, the key factor is to increase the balance accuracy, which is improving rotor efficiency, reliability and safety. So, it is necessary to measure its amplitude and phase unbalance when the rotor is rotating, and correct its unbalance amount.

For the balance measuring systems, many scholars have proposed a series of excellent correlation algorithms. Reference [2] proposed unbalance signal processing method based on residuals MUSIC spectral analysis. Reference [3] proposed an adaptive filtering method to extract useful signals information feature in strong noise background. These algorithms have good efficiency for signals processing in software, but they need complex hardware design. Currently, most electric measure systems are component of single-chip microcontroller and a variety of peripheral devices. This cascade formation increases the near-frequency signal effect of measuring channel. And it also takes some disadvantages, such as low integration, strong interference and low running speed. Based on analysis above, we improve the balancing machine electric measure systems from two points in this paper:

(1) For decreasing complexity of hardware design, our system use dual-core OMAP embedded system as the microcontroller. The system integrates ARM and DSP, and it can reflect strong control ability and fast information processing ability.

(2) For decreasing near-frequency signals effect of measurement channel, we achieve the digital band-pass tracking filter based on the harmonic wavelet packet algorithm. Because the harmonic wavelet packet in the frequency domain has the characteristics of subdividing the signal infinitely, local features of weak signals can be analyzed and extracted.

\section{EMBEDDED BALANCE MEASUREMENT SYSTEM STRUCTURE AND PRINCIPLE BASED ON OMAP}

\section{A. The Overall Structure of the Electrical Measurement System}

The function of electrical measurement system is to implement pre-processing weak signals and extract unbalance rapidly, which they have the same frequencies compared with rotor noises from sensors. And then it obtains the amplitude and phase unbalances information, completes the milling. Electrical measuring system structure is shown in Fig.1. The system includes preamble signal processing circuit, A/D transformation circuit, the digital band-pass tracking filter based on the harmonic wavelet packet and OMAP embedded system [4-5].

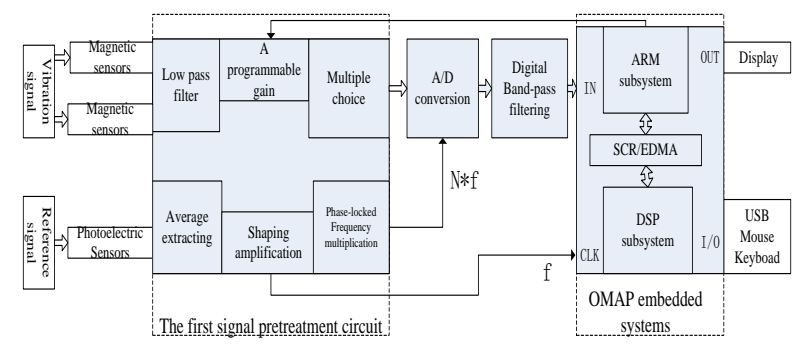

FIGURE I. ELECTRICAL MEASURING SYSTEM OVERALL STRUCTURE.

\section{B. Electrical Measuring System}

The system processes two-way signal in parallel. One is voltage signal that is switched by vibrating signals into, which 
is detected by left and right magneto electric sensors. Weak analog voltage signal is transmitted to the analog front-end circuit unit, and after analog circuit unit acquisition, signal amplification, low-pass filtering and programmable gain adjustment, the signal is transmitted next signal processing unit. Low-pass filtering circuit filters out high frequency components, reserves fundamental frequency information including unbalance data and other interference information. Programmable gain adjusts the signal circuitry within the range suitable for subsequent processing. The other is the reference signal that generated by photoelectric encoder. Analog frontend circuit unit is responsible for processing turn signals, which includes mean extraction, shaping amplification, frequency multiplier phase-locked. After that, this signal is used as time signal of A/D transformation.

After pre-process, two vibration signals have been the digital signals that is implemented $\mathrm{N}$ frequency multiplication clock signal processing. Two digital signals include lowfrequency clutter interference, especially near-frequency signal. After processing above, the signals are transmitted to OMAP embedded system.

\section{DIGITAL FILTERING PRINCIPLES BASED ON HARMONIC WAVELET PACKET AND REALIZATION}

\section{A. Electrical Measuring System Disturbances}

Balancing test systems work environment subject to various interference sources affect. It mainly includes[6] cochannel interference, high-frequency interference, nearfrequency interference. It is hard to distinguish co-channel interference that caused by unwanted signal carrier frequency and the same the carrier frequency of rotation spindle. So it needs to decrease interference by optimizing hardware design and repeating calibration. The high-frequency interference caused by natural frequency of the mechanical system and drive system, is solved by pre-low-pass filter. However, the near-frequency interference is caused by noise tolerance, temperature, and humidity of simulation electronic components. It is the huge challenge compared with other two interferences.

\section{B. Armonic Wavelet Packet Transforms}

$x(t)$ is Continuous-time signal, its transformation in the scale parameter $m 、 n$, and its Corresponding harmonic wavelet transform as shown in (1),

$$
W_{x}(m, n, k)=(n-m) \int_{-\infty}^{+\infty} x(t) \bar{\psi}\left(t-\frac{k}{n-m}\right) d t
$$

After the Fourier transform, we can have

$$
W_{x}(m, n, w)=X(w) \bar{\psi}_{m, n}[(n-m) w]
$$

Its discrete form can be written as

$$
W_{x}(m, n, k)=\frac{n-m}{N} \sum_{R=0}^{n-1} x(r) \bar{\psi}\left(r-\frac{k}{n-m}\right) \quad(r=1,2,3 \cdots N-1)
$$

Although the spectral characteristics of the harmonic wavelet have the advantages of compact, precise positioning capability phase, function expressions clear, realizing the algorithm easily, harmonic wavelet cannot do any detailed description of the local signal the band during analysis. As the extension of harmonic wavelet, based on excellent characteristics of harmonic wavelet basis, harmonic wavelet packet can be realized refinement infinitely on any frequency band signals. In this paper, digital band-pass tracking filter is designed by software based on harmonic wavelet packet transform [7]. We obtain the unbalance signal by harmonic wavelet package subdivision and digital filter tracking characteristics [8]. Harmonic wavelet packet frequency-domain distribution is shown below.

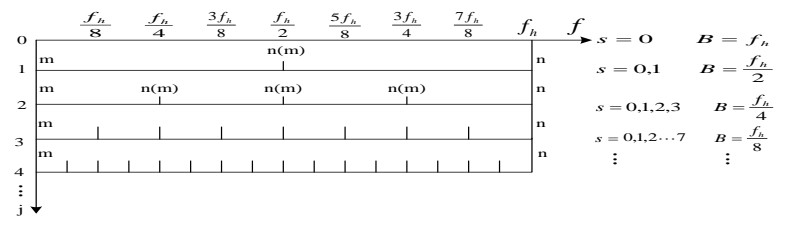

\section{FIGURE II. DISTRIBUTION FREQUENCY DOMAIN HARMONIC WAVELET PACKET DECOMPOSITION.}

From Fig.2, signal can be decomposed to the corresponding layer to extract required information frequency characteristic. By selecting the appropriate decomposition level $j$ and the number of bands $S$, we have observed detail features of the signal better. The signal frequency bandwidth $B$, the limit $m$ and $n$ is determined by the following equations.

$$
\begin{aligned}
B= & 2^{-j} f_{h} \\
& \left\{\begin{array}{c}
m=s B \\
n=(s+1) B
\end{array} \quad s=0,1 \cdots 2^{-j}-1 .\right.
\end{aligned}
$$

In the equations (4), $f_{h}$ is the highest observed frequency, $s$ is band value. When $j$ is increasing, observed signal tends to high-frequency. Therefore, in harmonic signal wavelet packet analysis, it is the primary task that selects the appropriate decomposition level and frequency values.

According to the principle of harmonic wavelet packet transform, digital filtering step that filtering near frequency are as follows:

(1) According to the characteristics of vibration signals of measuring system to determine the harmonic wavelet packet decomposition scale factor $j$ and the band number $S$.

(2) From equation (4), calculate Frequency-domain bandwidth $B$ of harmonic wavelet packet.

(3) Doing fast Fourier transform to the discrete time series signal, and calculate the discrete values of the frequency domain.

(4) From equation (5), calculate Wavelet Packet upper and lower limits in accuracy the band, complete frequency domain analysis.

(5) Inverse FFT, calculate reconstructed time domain signal after wavelet packet transform, as determined by the signal band to extract time-domain waveform. 


\section{Anti-near-frequency Harmonic Wavelet Packet Filter Design}

Unbalance vibration signals are usually very weak and drowned themselves in the noise. Harmonic wavelet packet digital tracking filter is shown in Fig.3.

Fig.3(a) is the original vibration signal waveforms (including unbalanced signals) in the scene of the rotor speed of 16000RPM. As can be seen from the Fig.3(a), although the vibration signal is a periodic sinusoidal signal, unbalanced signals are drowned in noise and cannot be distinguished.

After pre-pretreated low-pass filter, the time domain signal is shown in Fig.3 (b). Original signal mutation frequency component is filtered out basically, signal becomes smooth and chiseled. But unbalanced signal is still included in other lowfrequency signal which is still not effectively extract the information characteristics of baseband signal.

Set sampling frequency $f_{s}=512 \mathrm{~Hz}$ and analysis frequency $f_{h}=30 \mathrm{~Hz}$, the low-pass time-domain signal is transformed with FFT, and then spectrum is shown in Fig.3(c). Because of the strong low-frequency interference of the fundamental frequency signal in the frequency domain, the base frequency of the frequency signal is almost not distinguish clearly, so, it needs further processing in frequency domain .

We use harmonic wavelet and harmonic wavelet packet to decompose and extract the frequency domain signals. Signal band is set to 28-32Hz; spectrum is shown in Fig.3(d), which is after harmonic wavelet transformation. There is obviously effect of the other low frequency signal within the entire frequency band, and it is not achieved effectively suppress noise to the signal.

We use 'db6' decomposed signal, and set $\mathrm{n} 1=6, \mathrm{~B} 1=4 \mathrm{~Hz}$, and signal band are between $28-32 \mathrm{~Hz}$ and $496-500 \mathrm{~Hz}$. And then spectrum is shown in Fig.3(e). Compared with harmonic wavelet, the redundancy frequency components of signal spectrum that harmonic wavelet packet extracted reduces significantly. Especially for a certain frequency band near the frequency of the suppression signal has an excellent effect. After frequency domain signal reconstruction, we obtain signal time domain waveform of rotor baseband signal, as shown in Fig.3 (f).

Based on above process, we can conclude that the design have a good effect for the extraction of the amplitude and phase information.

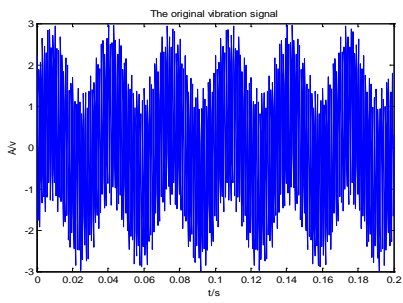

(a) Original rotor vibration signal

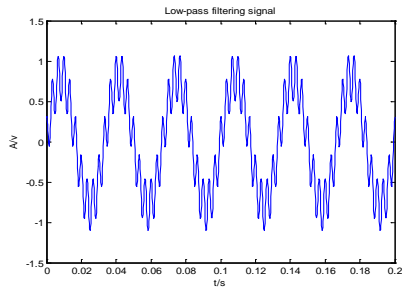

(b) Low pass filtering the signal

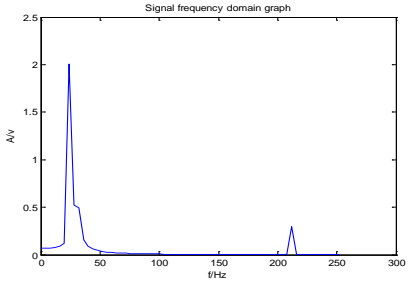

(c) Low pass filtering the signal spectrum spectrum spectrum

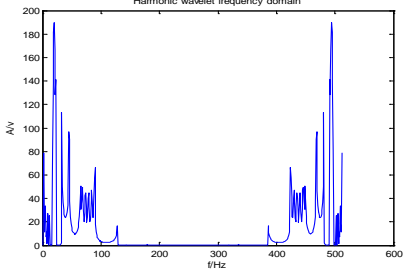

(d) Harmonic wavelet transform signal

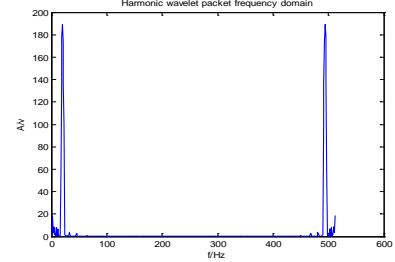

(e) Harmonic wavelet packet transform signal spectrum

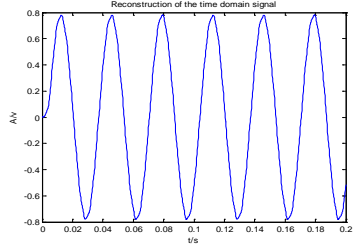

(f) Reconstruction of thefiltered time domain signal FIGURE III. ANTI NEARLY FREQUENCY HARMONIC WAVELET PACKET FILTER DESIGN.

\section{CONCLUSION}

In this paper, we present an embedded intelligent milling de-weight balance measurement system based on OMAP. This system can not only decrease the complexity of external circuit and external analogs, but also save the design cost. OMAP Dual-core architecture could design balance measurement system task reasonably according to their strengths. ARM system and DSP system are responsible for controlling and processing signals respectively. Simultaneously, software algorithm implement digital narrowband tracking filter based on harmonic wavelet packet. Experiments show that the filter 
could suppress near-frequency signal interference, and retain information characteristic of initial imbalance signal.

\section{ACKNOWLEDGEMENTS}

This work was supported by the Jilin Science and Technology Department Key Scientific and Technological Achievements Transformation Project (No. 20140307009GX).

\section{REFERENCES}

[1] Lees A W, Sinha J K, Friswell M I. Model-based identification of rotating machines [J]. Mechanical Systems and Signal Processing, 2009,23(6):1884-1893.

[2] ZHAO Dingding. High precision and high efficiency rigid support critical research techniques balancing machine measuring system [D]. $\mathrm{PhD}$ thesis, Shanghai Jiao tong University, 2013.01.

[3] LIU Jian, PAN Shuangxia, YANG Keji. Balancing Machines Filter Method study tracking digital [J]. Journal of Scientific Instrument, 2005,26(4):433-436.

[4] OMAP-L138 Applications Processor System Reference Guide. Texas Instruments, 2012.

[5] TMS320C674X/OMAP-L138 Processor External Memory Interface A (EMIFA) Users Guide. Texas Instruments, 2012.

[6] FENG Yingpeng. Vibration signal processing method to measure with precision balancing to achieve [D].Master thesis, Shanghai Normal University, 2013.05.

[7] John W. Kelly et al. "Frequency Tracking and Variable Bandwidth for Line Noise Filtering without a Reference [J],” Annual International Conference of the IEEE, pp.7908-7911, 2011.

[8] G.B.LI, Y.H.LIN, H.ZH.WANG, et al. Harmonic Wavelet Packet Analysis of Friction-Induced Vibration [J]. Tribology Transactions, 2011,54(6):895-901. 